\title{
Two-dimensional vibronic spectroscopy of molecular aggregates: Trimers, dimers, and monomers
}

M. Keß, G. Worth, and V. Engel

Citation: The Journal of Chemical Physics 145, 084305 (2016); doi: 10.1063/1.4961388

View online: http://dx.doi.org/10.1063/1.4961388

View Table of Contents: http://aip.scitation.org/toc/jcp/145/8

Published by the American Institute of Physics

\section{Articles you may be interested in}

Electron-nuclear wave-packet dynamics through a conical intersection

The Journal of Chemical Physics 146, 074304 (2017); 10.1063/1.4975811

Communication: Adiabatic and non-adiabatic electron-nuclear motion: Quantum and classical dynamics The Journal of Chemical Physics 144, 171103 (2016); 10.1063/1.4948777

Two-dimensional electronic spectroscopy can fully characterize the population transfer in molecular systems The Journal of Chemical Physics 145, 124312 (2016); 10.1063/1.4962577

Two-dimensional Fourier transform electronic spectroscopy

The Journal of Chemical Physics 115, 6606 (2001); 10.1063/1.1398579

Vibronic coupling explains the ultrafast carotenoid-to-bacteriochlorophyll energy transfer in natural and artificial light harvesters

The Journal of Chemical Physics 142, 212434 (2015); 10.1063/1.4919548

Optimizing sparse sampling for 2D electronic spectroscopy

The Journal of Chemical Physics 146, 084201 (2017); 10.1063/1.4976309

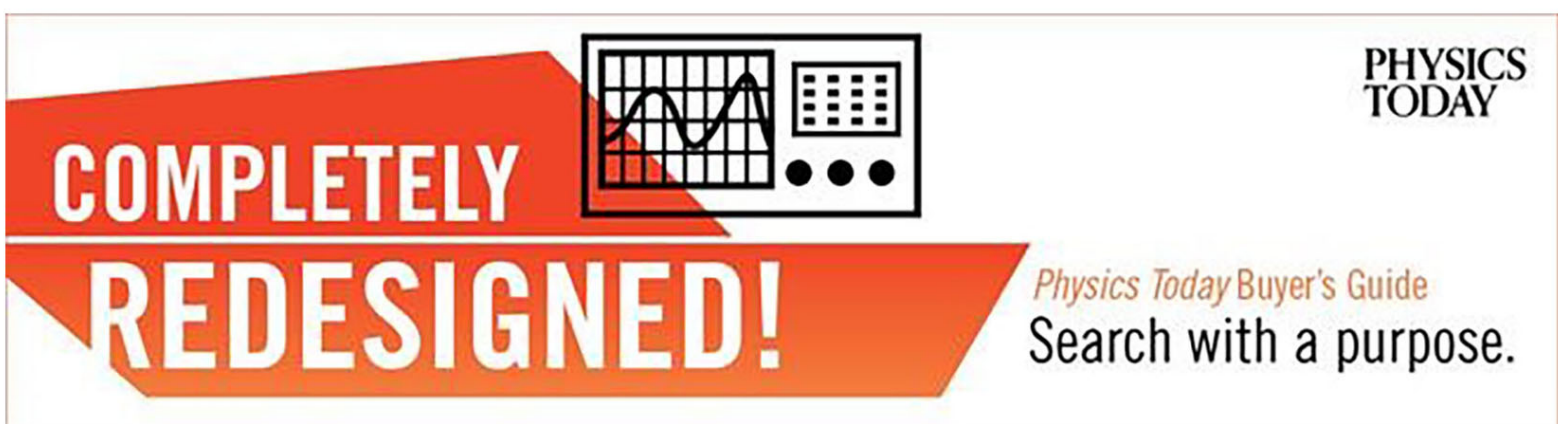




\title{
Two-dimensional vibronic spectroscopy of molecular aggregates: Trimers, dimers, and monomers
}

\author{
M. Keß, ${ }^{1}$ G. Worth, ${ }^{2}$ and V. Engel ${ }^{1}$ \\ ${ }^{1}$ Institut für Physikalische und Theoretische Chemie, Universität Würzburg, Campus Nord, \\ Emil-Fischer-Str. 42, 97074 Würzburg, Germany \\ ${ }^{2}$ School of Chemistry, University of Birmingham, Edgbaston, Birmingham B15 2TT, United Kingdom
}

(Received 13 June 2016; accepted 8 August 2016; published online 26 August 2016)

\begin{abstract}
The two-dimensional (2D) vibronic spectroscopy of molecular trimers is studied theoretically. The solution of the time-dependent Schrödinger equation is carried out with the multi-configurational time-dependent Hartree (MCTDH) method which allows for an efficient propagation of the multicomponent wave functions. 2D-spectra are calculated for $\mathrm{H}$ - and J-type aggregates incorporating one or two vibrational modes for each monomer. In performing calculations for monomer, dimer, and trimer systems, it is documented how the vibronic structure of the 2D-spectrum changes upon aggregation. This is of importance for the characterization of aggregation behavior being influenced by experimental conditions such as temperature or concentration. Published by AIP Publishing. [http://dx.doi.org/10.1063/1.4961388]
\end{abstract}

\section{INTRODUCTION}

Ever since the early work of Jelly ${ }^{1}$ and Scheibe $^{2}$ molecular aggregates have attracted much attention. Besides their interesting optical properties ${ }^{3}$ such systems are of basic importance to understand photosynthesis ${ }^{4}$ and the efficiency of organic photovoltaic devices. ${ }^{5-7}$ From a theoretical point of view, exciton theory ${ }^{8-10}$ is the starting point to arrive at a realistic description of, e.g., the optical spectroscopy of molecular aggregates. In an extension of models involving only electronic levels, the role of vibrations has been addressed very early, ${ }^{11-13}$ and a thorough numerical example was given for the vibronic dimer by Fulton and Gouterman. ${ }^{14,15}$ For a comprehensive review on the vibronic theory of aggregates see the recent work by Schröter et al. ${ }^{16}$ An essential quantity is the magnitude of the electronic matrix element which couples excitonic states in an aggregate, ${ }^{10,16}$ and two-dimensional (2D) spectroscopic methods provide a means to measure this coupling. Originating from nuclear magnetic resonance spectroscopy, ${ }^{17,18}$ such techniques have been successfully applied in the infrared ${ }^{19-24}$ and also in the optical region. ${ }^{25-29}$ Recent applications of 2D optical spectroscopy can be found, e.g., in Refs. 30-45.

Calculations of 2D-spectra are most commonly performed using density-matrix theory. ${ }^{46-53}$ A wave-function based description is also possible ${ }^{54-56}$ but system-bath couplings are difficult to include within this approach. However, it was shown recently that 2D-spectra, within several approximations, can be efficiently calculated employing stochastic Schrödinger equations. ${ }^{57,58}$ In this paper we use a wave-function description to arrive at 2D-spectra and neglect all couplings to a surrounding.

The aggregate Hamiltonians usually employed use internal monomer vibrational degrees of freedom, ${ }^{10,16,59,60}$ but it should be kept in mind that intermolecular degrees of freedom cannot always be ignored. ${ }^{61,62}$ To estimate the computational effort to describe the dynamics of an aggregate coupled to laser fields as used in 2D photon-echo experiments, let us regard the molecular trimer (M-M-M) with only a single vibrational coordinate $x_{n}$ per monomer $(n)$. Such single mode models have been applied to many aggregates ${ }^{16,60}$ and the role of single- versus multi-mode models was analyzed in Ref. 63. Besides the electronic ground state, one has to incorporate three singly excited configurations (( $\left.\mathrm{M}^{*}-\mathrm{M}-\mathrm{M}\right),\left(\mathrm{M}-\mathrm{M}^{*}-\mathrm{M}\right)$, and $\left(\mathrm{M}-\mathrm{M}-\mathrm{M}^{*}\right)$ ) where any one of the three monomers $\mathrm{M}$ is excited upon photon absorption. These states have different potential energy surfaces $V_{e n}\left(x_{1}, x_{2}, x_{3}\right)$ and are coupled by off-diagonal potential matrix elements, i.e., the electronic coupling mentioned above. Furthermore, coupled doubly excited states $\left(\left(M^{*}-M^{*}-M\right),\left(M^{*}-M-M^{*}\right),\left(M-M^{*}-M^{*}\right)\right)$ have to be taken into account. Adding the field-matter interaction we thus are faced with the problem of 7 coupled electronic states, where the dimension of the intramolecular motion is three.

To obtain a 2D-spectrum, the third-order polarization has to be calculated which involves loops over two timevariables, usually being a pulse delay $\tau$ and the detection time $t^{\prime}$, see Sec. II A. It is clear that this involves a large computational effort and one needs an efficient method for the solution of the time-dependent Schrödinger equation. Fortunately, the multi-configurational time-dependent Hartree (MCTDH) method ${ }^{64-66}$ provides such a tool. It is then possible to calculate the time-dependent polarization induced by the external fields. However, this observable contains terms in all orders of the field-matter interaction and signal fields emitted in many different directions. Because in a photon-echo 2D-arrangement the signal is detected under phase-matching conditions (see, however, the "phase-cycling" arrangement where a fluorescence signal is detected ${ }^{67,68}$ ) one needs to extract a signal field in the phase-matching direction. This can be achieved by performing several calculations with phase tagged electric fields, ${ }^{69,70}$ for an alternative method see Refs. 50 and 71. 
Within a liquid, the self-assembly of molecules critically depends on experimental parameters such as concentration and temperature. As an example, aggregates of perylene bisimides were studied under varying experimental conditions. ${ }^{72}$ In comparing absorption ${ }^{73}$ and circular dichroism spectra $^{74}$ measured at different temperatures and calculated for different aggregate sizes, information on the average aggregate size could be retrieved. In this paper we address the question of how 2D-spectra change upon aggregation where a focus is on the influence of vibrational motion. In Sec. II we briefly introduce the method to calculate the polarization under phase-matching conditions and also provide details of the employed model. Trimer spectra calculated for different geometries, incorporating one or two vibrational degrees of freedom per monomer, are presented in Sec. III. There, we also compare spectra of monomer, dimer, and trimer systems as well as those arising from a mixture of such species. A summary of the results is given in Sec. IV.

\section{THEORY}

\section{A. Calculation of 2D-spectra}

Two-dimensional optical spectra result from the dipole interaction of molecular samples with three laser pulses described by the electric field

$\vec{E}(t)=\vec{\epsilon} E(t)=\vec{\epsilon} \sum_{n=1}^{3} g_{n}\left(t-T_{n}\right) \cos \left[\omega_{n}\left(t-T_{n}\right)-\phi_{n, s}\right]$.

Here, the polarization vectors of all fields are taken as equal $\left(\vec{\epsilon}_{n}=\vec{\epsilon}\right)$ and $\omega_{n}$ are the laser frequencies. The envelope functions $g_{n}\left(t-T_{n}\right)$ are centered at times $T_{n}$. The delay time $\tau=T_{2}-T_{1}$ is defined as the time difference between the first and the second pulse, and the detection time $t^{\prime}=t-T_{3}$ starts at the center of the third pulse. Because we here exclude couplings to a surrounding, it is sufficient to treat the case of zero population time, ${ }^{49}$ e.g., we set $T=T_{3}-T_{2}=0$. The phases $\phi_{n, s}$ are specified below. In our calculations we determine the projection of the third-order polarization on $\vec{\epsilon}$.

The central quantity in the calculation of $2 \mathrm{D}$-spectra is the third-order polarization emitted along the direction $\vec{k}_{s}$ $=-\vec{k}_{1}+\vec{k}_{2}+\vec{k}_{3}$ (phase-matching), where the $\vec{k}_{n}(n=1,2,3)$ denote the wave vectors of the three laser fields. We employ a non-perturbative scheme ${ }^{69,70}$ where first the overall polarization, defined as the expectation value of the projection $\mu=\vec{\mu} \vec{\epsilon}$ of the transition dipole moment $\vec{\mu}$ on $\vec{\epsilon}$ (containing all orders and directions), is calculated and then the contribution emitted along the direction $\vec{k}_{s}$ is extracted. Under several assumptions, the number of possible directions can be limited to $12 .^{70}$ Decomposing the polarization $P(\vec{\Phi})$ into a Fourierseries with these 12 contributions yields (omitting the timearguments)

$$
P(\vec{\Phi})=\sum_{q=1}^{12} e^{i\left(n_{q} \vec{k}_{1}+m_{q} \vec{k}_{2}+l_{q} \vec{k}_{3}\right) \vec{x}} P_{q}=\sum_{q=1}^{12} e^{i \vec{r}_{q} \vec{\Phi}_{s}} P_{q},
$$

where $\vec{r}_{q}=\left(n_{q}, m_{q}, l_{q}\right)$ runs over the possible combinations of $(n, m, l)$, and $\vec{\Phi}_{s}=\left(\phi_{1, s}, \phi_{2, s}, \phi_{3, s}\right)$ are fixed phases.
To disentangle the directional terms and calculate the polarizations $P_{q}$ emitted in the different directions, one performs calculations of the polarization for chosen phases

$$
\begin{aligned}
P\left(\tau, t^{\prime}, \vec{\Phi}_{s}\right) & =\left\langle\psi\left(\tau, t^{\prime}, \vec{\Phi}_{s}\right)|\mu| \psi\left(\tau, t^{\prime}, \vec{\Phi}_{s}\right)\right\rangle \\
& =\tilde{P}\left(\tau, t^{\prime}, \vec{\Phi}_{s}\right)+\tilde{P}^{*}\left(\tau, t^{\prime}, \vec{\Phi}_{s}\right) .
\end{aligned}
$$

The wave functions $\psi\left(\tau, t^{\prime}, \vec{\Phi}_{s}\right)$ are obtained as solutions of the time-dependent Schrödinger equation for the system coupled to the laser fields. Repeating the calculation for 12 combinations of phases leads to the linear system of equations

$$
\left(\begin{array}{cccc}
c_{1,1} & c_{1,2} & \cdots & c_{1,12} \\
c_{2,1} & \ddots & & \vdots \\
\vdots & & \ddots & \vdots \\
c_{12,1} & \cdots & \cdots & c_{12,12}
\end{array}\right)\left(\begin{array}{c}
P_{1} \\
P_{2} \\
\vdots \\
P_{12}
\end{array}\right)=\left(\begin{array}{c}
\tilde{P}\left(\vec{\Phi}_{1}\right) \\
\tilde{P}\left(\vec{\Phi}_{2}\right) \\
\vdots \\
\tilde{P}\left(\vec{\Phi}_{12}\right)
\end{array}\right)
$$

with coefficients $c_{s, q}=e^{i \vec{r}_{q} \vec{\Phi}_{s}}$. Details of the procedure and the explicit values for the wave vectors and phases are given in Ref. 70.

A Fourier transform of the component $P_{q}\left(\tau, t^{\prime}\right)$ of the third order polarization which is emitted in the $\vec{k}_{s}$ direction leads to the $2 \mathrm{D}$ spectrum ${ }^{75}$

$S_{q}\left(E_{\tau}, E_{t^{\prime}}\right)=\frac{i}{(2 \pi)^{2}} \int d \tau \int d t^{\prime} e^{-i E_{\tau} \tau} e^{i E_{t^{\prime} t^{\prime}}} P_{q}\left(\tau, t^{\prime}\right)$.

In calculating the spectra presented in Sec. III, the polarization is convoluted with Gaussians having a width (full width at half-maximum) of $110 \mathrm{fs}$ (spectral resolution of $0.033 \mathrm{eV}$ ) in both directions. This means that the sampled time intervals along $\tau$ and $t^{\prime}$ can be chosen as short as $200 \mathrm{fs}$, where we use a time step of 0.8 fs. To obtain smoother spectra, the polarization is filled with zeros for times larger than $200 \mathrm{fs}$ before Fourier transforming. If not noted differently, the spectra discussed below are normalized to their largest peak and the real (absorptive) part is shown.

\section{B. Model}

The aggregate model builds on monomer units having two electronic states $|g\rangle$ and $|e\rangle$ coupled to a vibrational degree of freedom along the dimensionless coordinate $x$. The ground and excited state vibrational Hamiltonians are

$$
\begin{aligned}
& H_{g}(x)=-\frac{1}{2} \frac{d^{2}}{d x^{2}}+\frac{1}{2} \omega_{v i b}^{2} x^{2}, \\
& H_{e}(x)=-\frac{1}{2} \frac{d^{2}}{d x^{2}}+\frac{1}{2} \omega_{v i b}^{2}\left(x-x_{e}\right)^{2}+\Delta E .
\end{aligned}
$$

The vibrational frequency $\omega_{v i b}$ is assumed to be identical in the ground and excited state and is assigned a value of $\omega_{v i b}=0.175 \mathrm{eV}$. The displacement in equilibrium position is taken as $x_{e}=2.57(\mathrm{eV})^{-1 / 2}$. These parameters stem from our calculation on perylene bisimide aggregates. ${ }^{76}$ The energy shift is taken as $\Delta E=1.0 \mathrm{eV}$. 
The monomer (M) Hamiltonian including the field-matter interaction reads

$$
H^{M}(x)=\left(\begin{array}{cc}
H_{g} & W(t) \\
W(t) & H_{e}
\end{array}\right),
$$

with the time-dependent interaction

$$
W(t)=-\mu E(t) .
$$

The magnitude of $\mu$ is set to a value of one in all calculations.

The dimer Hamiltonian is constructed from two monomer Hamiltonians and contains four electronic states. Photoabsorption from the ground state $|g(1), g(2)\rangle=|g g\rangle$ leads to the population of singly excited states $|e g\rangle$ (first monomer excited) and $|g e\rangle$ (second monomer excited). These two states are electronically coupled by a constant coupling element $J$ which we fix to be positive and having the value of $0.25 \mathrm{eV}$. Finally, the doubly excited state $|e e\rangle$ represents an excitation of both monomers. Using the shorthand notation

$$
H_{s_{1} s_{2}}=H_{s_{1}}\left(x_{1}\right)+H_{s_{2}}\left(x_{2}\right), \quad\left(s_{1}, s_{2}\right) \epsilon(g, e),
$$

where $x_{n}$ is the vibrational mode of monomer $(n)$, we obtain the dimer Hamiltonian as

$$
H^{D}=\left(\begin{array}{cccc}
H_{g g} & W(1) & W(2) & 0 \\
W(1) & H_{e g} & J & W(2) \\
W(2) & J & H_{g e} & W(1) \\
0 & W(2) & W(1) & H_{e e}
\end{array}\right) .
$$

The system-field-interaction $W(n)$ leading to excitation of monomer $(n)$ is

$$
\begin{aligned}
& W(1)=-\mu E(t) f_{+}(\gamma), \\
& W(2)=-\mu E(t) f_{-}(\gamma) .
\end{aligned}
$$

Here appear the geometry factors $f_{ \pm}(\gamma)$ which depend on the orientation angle between the two monomer transition dipole-moments. We use the fixed configuration where the monomer transition dipole vectors $\vec{\mu}_{n}$ lie in the $(x, y)$-plane with the $y$-axis intersecting the angle $\gamma$ between them. The motivation to use this particular geometry is that, for onephoton absorption, the absorption spectrum calculated within this arrangement is identical (within an overall factor) to the one obtained if the orientational average is performed ${ }^{77}$ which, however, is not true for a $2 \mathrm{D}$-spectrum ${ }^{51}$ An average over molecular orientations results in different intensities of certain peaks appearing in the spectra. This is not essential for the present investigation and we restrict the calculations to the fixed molecular orientation. For an $(x, y)$-polarized electric field with polarization vector $\vec{\epsilon}=(1,1,0)$ one finds $^{78}$

$$
f_{ \pm}(\gamma)=\cos \frac{\gamma}{2} \pm \sin \frac{\gamma}{2}
$$

Most of our considerations are restricted to parallel $\left(\gamma=0^{\circ}\right)$ and antiparallel $\left(\gamma=180^{\circ}\right)$ dipole geometries. This, because we fix the excited state coupling to a positive number, corresponds to the case of a $\mathrm{H}$ - and J-aggregate, respectively. For a J-aggregate (named after Jelly ${ }^{1}$ ), the one-photon absorption from the ground state leads to excitation of the lowest band of the excitonic manifold. On the other hand, for the $\gamma=0^{\circ}$ geometry, the absorption band of an aggregate shows a hypsochromic $(\mathrm{H})$ shift with respect to the monomer absorption.

The trimer Hamiltonian contains, besides the common ground state, three singly and three doubly excited states of the form $\left|s_{1} s_{2} s_{3}\right\rangle=\left|s_{1}(1), s_{2}(2), s_{3}(3)\right\rangle$ with the corresponding vibrational Hamiltonians

$$
H_{s_{1} s_{2} s_{3}}=H_{s_{1}}\left(x_{1}\right)+H_{s_{2}}\left(x_{2}\right)+H_{s_{3}}\left(x_{3}\right), \quad\left(s_{1}, s_{2}, s_{3}\right) \epsilon(g, e) \text {. }
$$

Note that the triply excited state $|e e e\rangle$ does not need to be included because the resulting signal is not emitted along the phase-matching direction. Each singly excited state possesses two monomeric units in the ground state, and in the doubly excited states only one monomer remains in the ground state. One-photon transitions couple the manifold of singly excited states with the ground state and the singly excited states with the three doubly excited states. Here we only regard trimers with next-neighbor couplings excluding the case of cyclic geometries where the first and last monomers are coupled. The trimer Hamiltonian then reads

$$
H^{T}=\left(\begin{array}{ccccccc}
H_{g g g} & W(1) & W(2) & W(3) & 0 & 0 & 0 \\
W(1) & H_{\text {egg }} & J & 0 & W(2) & W(3) & 0 \\
W(2) & J & H_{\text {geg }} & J & W(1) & 0 & W(3) \\
W(3) & 0 & J & H_{\text {gge }} & 0 & W(1) & W(2) \\
0 & W(2) & W(1) & 0 & H_{\text {eeg }} & J & 0 \\
0 & W(3) & 0 & W(1) & J & H_{\text {ege }} & J \\
0 & 0 & W(3) & W(2) & 0 & J & H_{\text {gee }}
\end{array}\right) .
$$

In our calculations, we assume that the couplings between the singly excited states and between the doubly excited states are identical. The fixed orientation of the monomeric transition dipole-moments is treated in a similar way as for the dimer. ${ }^{79}$ Within the planar geometry $\vec{\mu}_{2}$ points along the $y$-axis and the moments $\vec{\mu}_{1}$ and $\vec{\mu}_{3}$ are rotated by angles $\mp \gamma$ with respect to $\vec{\mu}_{2}$. This leads to the interaction terms

$$
W(m)=-\mu E(t) f_{m}(\gamma),
$$

with the angular factors

$$
f_{1}(\gamma)=\cos \gamma+\sin \gamma, f_{2}(\gamma)=1, f_{3}(\gamma)=\cos \gamma-\sin \gamma .
$$

For comparison, we also regard purely electronic systems. In these cases, the monomer vibrational Hamiltonians are numbers, i.e., $H_{g}(x)=0$ and $H_{e}(x)=\Delta E$, respectively. Then, the dimer and trimer problems reduce to those of coupled 4-level and 7-level systems, respectively.

The field strengths are taken such that the maximal value of the dipole coupling $\left(\mu g_{n}(0)\right)$ is $0.03 \mathrm{eV}$. This corresponds to a field strength (for our value of $\mu=1$ a.u.) of about $3.5 \times 10^{10} \mathrm{~W} / \mathrm{cm}^{2}$. It is checked numerically that for this coupling the contributions to the polarization having orders higher than three are negligible. The pulse-envelope functions 
$g_{n}$ are Gaussians with a temporal width of 5.4 fs. The photon energies of all pulses are equal and have values of $\omega=1.0 \mathrm{eV}$ (electronic problem) and $\omega=1.15 \mathrm{eV}$ (vibronic problem), respectively. This leads to resonant transitions between the involved states. As initial state, the ground state is used in each case.

The wave packet propagation is carried out via the MCTDH-method using the Heidelberg package. ${ }^{80}$ This expands the nuclear wavepacket in sets of low-dimensional time-dependent basis functions, known as single particle functions (SPFs). These in turn are expanded on a primitive grid defined by a discrete variable representation (DVR). The SPFs and expansion coefficients then evolve in time according to equations of motion that provide a variational solution to the time-dependent Schrödinger equation. Each vibrational degree of freedom is treated with a harmonic oscillator DVR primitive basis with 15 grid points. The same number of time-dependent single particle functions (SPFs) are used for each degree of freedom, chosen so as to converge the spectrum obtained. For the dimer case 3,5,5,4 SPFs are used for the first, second, third, and fourth electronic state, respectively. For the trimer, a total number of 3 SPFs for the ground state and 5 SPFs for each of the six excited states are employed. The time-evolution of the monomer is treated using a full numerical solution of the time-dependent Schrödinger equation on the $1 \mathrm{D}$ primitive grid.

\section{RESULTS}

We first consider 2D-spectra for the purely electronic trimer system. Figure 1 illustrates the energy-level scheme where the left part refers to the parallel and the right part to the anti-parallel geometry. Besides the ground state at $E_{0}=0$,

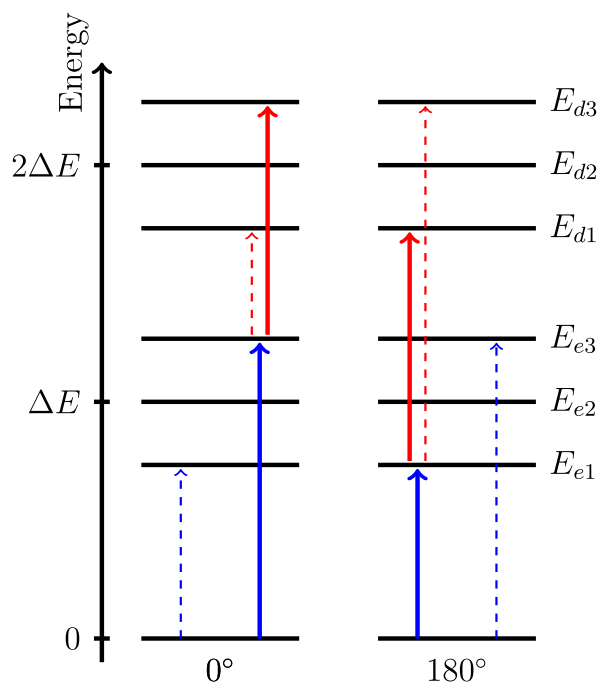

FIG. 1. Electronic level scheme for non-cyclic trimers with next-neighbor coupling. The ground state is at an energy of $E_{0}=0$ and the monomer excitation energy is $\Delta E$. Three singly and three doubly excited states are marked having energies $\left(E_{e 1}, E_{e 2}, E_{e 3}\right)$ and $\left(E_{d 1}, E_{d 2}, E_{d 3}\right)$, respectively. In the parallel transition dipole geometry $\left(\gamma=0^{\circ}, \mathrm{H}\right.$-aggregate $)$ transitions into the highest level of each band are favored (solid arrows). Weak transitions to the respective lowest states also occur (dashed arrows). This situation is reversed for the anti-parallel configuration at $\gamma=180^{\circ}$ (J-aggregate). three singly excited states with energies $E_{e 1}=\Delta E-\sqrt{2} J, E_{e 2}$ $=\Delta E$, and $E_{e 3}=\Delta E+\sqrt{2} J$ are present. ${ }^{81}$ The doubly excited states exhibit the same splitting and are located at energies $E_{d 1}=2 \Delta E-\sqrt{2} J, E_{d 2}=2 \Delta E$, and $E_{d 3}=2 \Delta E$ $+\sqrt{2} J$, respectively. In general, peaks in the 2D-spectra occur at positions which match energy differences $\left(E_{e n}-E_{0}\right)$ along $E_{\tau}$ and also differences $\left(E_{e n}-E_{0}\right)$ and $\left(E_{d m}-E_{e n}\right)$ along $E_{t^{\prime}} \cdot{ }^{55,82}$ For the given geometry, however, not all transitions are allowed. To anticipate which peaks are visible in the spectrum we regard the intensities $I_{n}$ of the absorption lines for transitions from the ground to the singly excited states with energies $E_{\text {en }}$. They are given, ignoring unimportant constants, as ${ }^{79}$

$$
\begin{aligned}
& I_{1}=1+\frac{1}{2} \cos (2 \gamma)-\sqrt{2} \cos (\gamma), \\
& I_{2}=1-\cos (2 \gamma), \\
& I_{3}=1+\frac{1}{2} \cos (2 \gamma)+\sqrt{2} \cos (\gamma) .
\end{aligned}
$$

For $\gamma=0^{\circ}$, one finds $I_{1}=3 / 2-\sqrt{2}, \quad I_{2}=0$, and $I_{3}$ $=3 / 2+\sqrt{2}$. Thus, besides a weak transition to the lowest excited state (for the positive value of $J$ as employed in our calculations), a strong transition to the upper level at $E_{e 3}$ takes place. Similar relative intensities are found for the transition from the singly excited states to the doubly excited states, as indicated by the arrows in Fig. 1. Using the numerical values of $\Delta E=1 \mathrm{eV}$ and $J=0.25 \mathrm{eV}$, it follows that an intense diagonal peak at $E_{\tau}=E_{t^{\prime}}=1.35 \mathrm{eV}$ and a single cross peak at $\left(E_{\tau}, E_{t^{\prime}}\right)=(1.35,1.0) \mathrm{eV}$ is expected to be seen in the $2 \mathrm{D}$-spectrum. This indeed is the case as can be verified by inspection of Fig. 2 (panel (a)) which shows the numerically determined real part of the spectrum. Changing the angle $\gamma$ to $180^{\circ}$ yields intensities of $I_{1}=3 / 2+\sqrt{2}, I_{2}=0$, and $I_{3}=3 / 2-\sqrt{2}$ so that transitions to the lower levels of the singly and doubly excited states are favored, see Fig. 1 . The peak positions are then calculated as $\left(E_{\tau}, E_{t^{\prime}}\right)=(0.65,0.65) \mathrm{eV}$ and $(0.65,1.0) \mathrm{eV}$, which is in accord with the numerically determined spectrum in Fig. 2 (panel (b)). Note that the peak structures of the off-diagonal peaks are different for the two geometries. This can be analyzed by regarding, within perturbation theory, the time-integrals appearing in the expressions for the polarization where the finite lengths of pulses enter. $^{54}$

We next turn to the vibronic 2D-spectra. Including a coupling between the three degenerate singly excited states $|e g g\rangle,|g e g\rangle,|g g e\rangle$ leads to a large density of vibrational states. The effect of vibronic coupling can already be seen in linear absorption spectra. ${ }^{73}$ Examples of vibronic 2Dspectra, which correspond to the geometries leading to the electronic spectra in Fig. 2, are shown in Fig. 3. As a general trend, a rich vibrational substructure is encountered. In a zeroth-order picture, we find vibronic progressions around the peak locations of the corresponding electronic spectra (Fig. 2). However, the coupling leads to a nonregular vibrational energy level spacing which is reflected in the 2D-spectrum of the H-aggregate (panel (a)). For example, it is seen that the (electronic) diagonal peak around $\left(E_{\tau}, E_{t^{\prime}}\right)=(1.45,1.45) \mathrm{eV}$ splits into several peaks. In the case of the J-aggregate (panel (b)) the appearance of the 


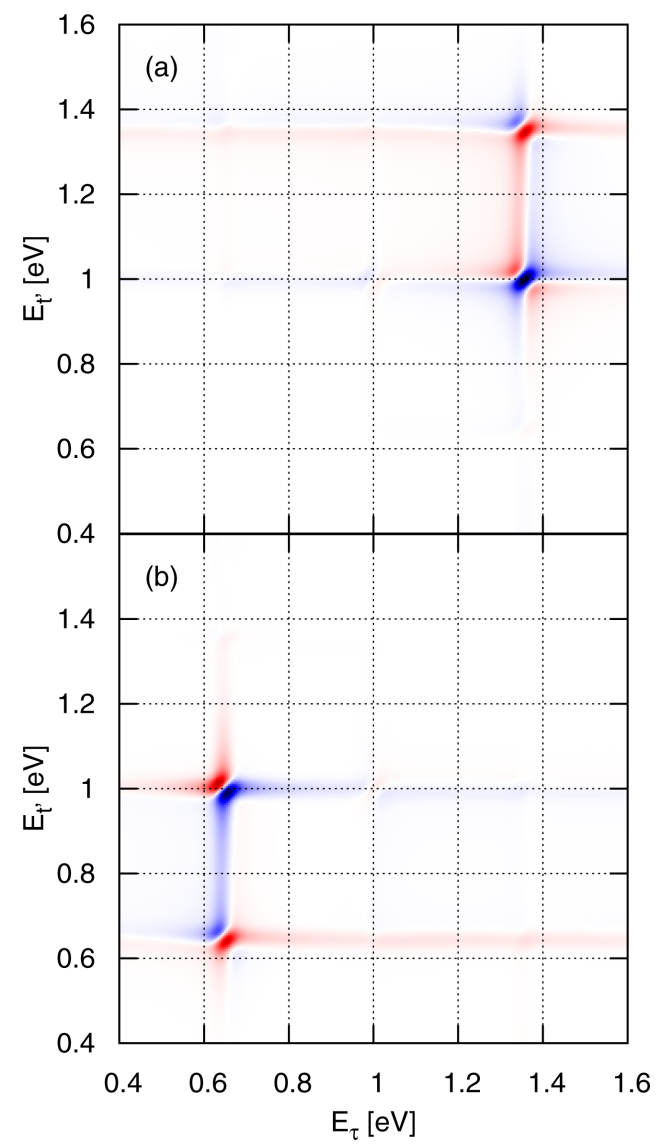

FIG. 2. Normalized 2D-spectra of the electronic trimer system. The case of an $\mathrm{H}$-aggregate $\left(\gamma=0^{\circ}\right.$, see Fig. 1) is depicted in panel (a) and the case of a J-aggregate $\left(\gamma=180^{\circ}\right.$, see Fig. 1) in panel (b). The color code is such that blue corresponds to positive and red to negative values.

spectrum is more regular. No peaks below $E_{\tau}=0.65 \mathrm{eV}$ are seen because, starting from the ground state, the lowest lying excited energy level is located at this energy. Along the energy $E_{t^{\prime}}$, peaks at lower energies are found because (stimulated emission) transitions from the excited state to higher lying vibrational levels in the ground state are possible. ${ }^{49}$ For our choice of parameters, the separation between electronic levels $(0.354 \mathrm{eV})$ is very close to twice the monomer vibrational level spacing $(0.350 \mathrm{eV})$. As a consequence, no clear assignment of peaks being associated with vibrational levels in a given electronically excited state is possible..$^{58}$

Until now, we regarded the cases of purely $\mathrm{H}-\left(\gamma=0^{\circ}\right)$ and J-aggregates $\left(\gamma=180^{\circ}\right)$. For a dipole-angle of $\gamma=90^{\circ}$, it is possible to excite all three singly excited states from the ground state so that a more structured spectrum is anticipated. Additionally, the sub-structure of the spectra will be influenced by the density of vibrational states. To give an example for such more complex situations, we regard the orthogonal dipole geometry and include a second monomer vibrational mode in the model so that the number of internal degrees of freedom is 6. The monomer Hamiltonians now are

$$
H_{g}(x, y)=-\frac{1}{2} \frac{\partial^{2}}{\partial x^{2}}-\frac{1}{2} \frac{\partial^{2}}{\partial y^{2}}+\frac{1}{2} \omega_{v i b, x}^{2} x^{2}+\frac{1}{2} \omega_{v i b, y}^{2} y^{2}
$$

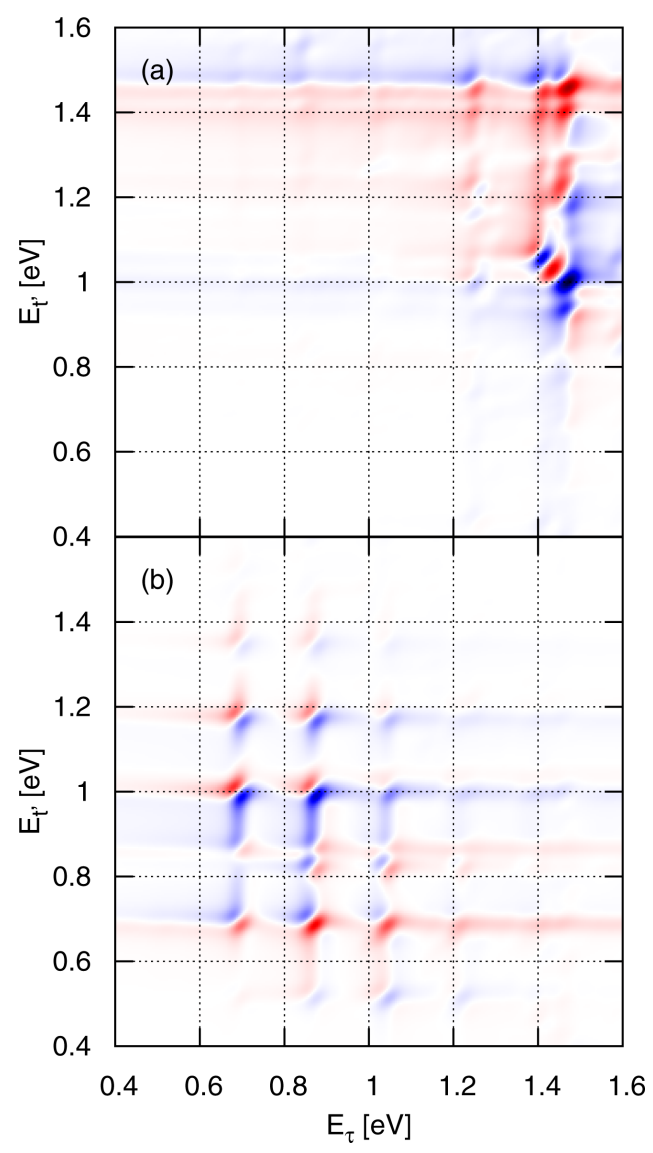

FIG. 3. Same as Fig. 2, but for the vibronic trimer system with one vibrational degree of freedom for each monomer.

$$
\begin{aligned}
H_{e}(x, y)= & -\frac{1}{2} \frac{\partial^{2}}{\partial x^{2}}-\frac{1}{2} \frac{\partial^{2}}{\partial y^{2}}+\frac{1}{2} \omega_{v i b, x}^{2}\left(x-x_{e}\right)^{2} \\
& +\frac{1}{2} \omega_{v i b, y}^{2}\left(y-y_{e}\right)^{2}+\Delta E_{e} .
\end{aligned}
$$

The parameters of the first oscillator (in $x$ ) are kept the same as before and the additional oscillator has the same excited state equilibrium distance $\left(y_{e}=x_{e}\right)$ but has a frequency of $\omega_{v i b, y}=0.120 \mathrm{eV}$. In Fig. 4, we compare 2D-spectra for the trimer with a single (panel (a)) and two (panel (b)) vibrational modes. For a better comparison, the spectra are not normalized. It is seen that the addition of the extra mode gives rise to a spectrum which is somehow less structured. A second vibronic progression leading to additional cross peaks is visible. However, unexpectedly, although the density of states increases in adding the extra oscillator, the appearance of the spectrum becomes simpler. This means that energetically close and thus overlapping peaks with finite widths are present so that certain features are averaged out.

We have shown that various effects influence the trimer $2 \mathrm{D}$-spectra. Among them are the vibrational degrees of freedom and the relative orientation of the monomer transition dipole-moments. It is interesting to regard the question of how 2D-spectra change upon aggregation. Therefore we compare monomer, dimer, and trimer spectra for the $\gamma=180^{\circ}$ geometry and the single monomer degree of freedom (using the same model parameters as before). The respective spectra are 


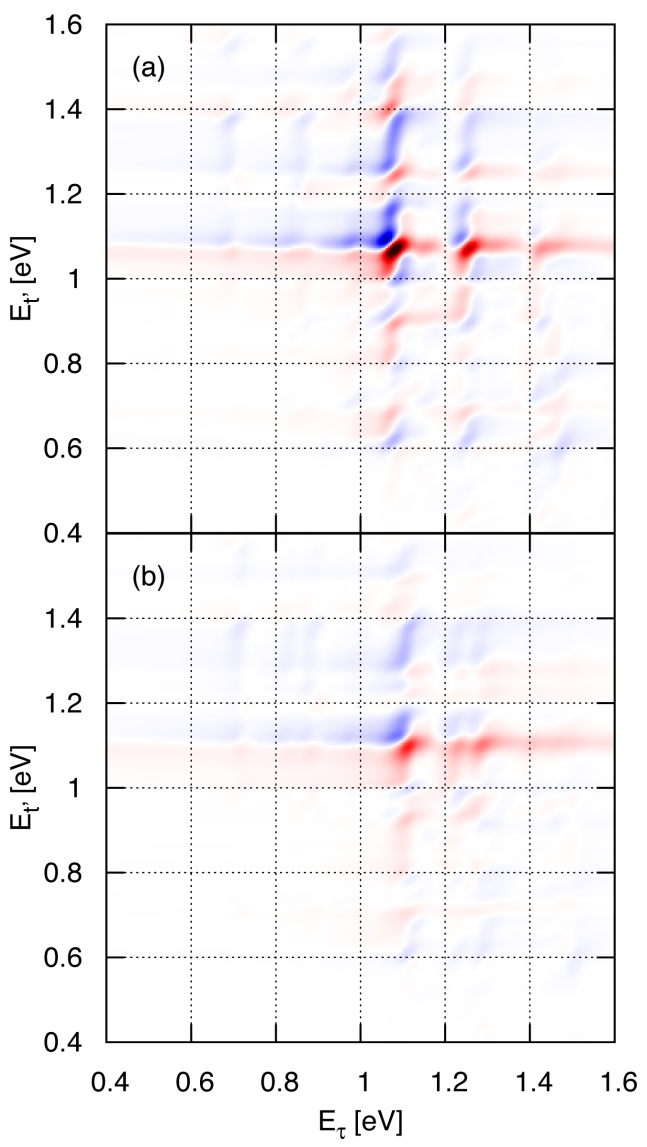

FIG. 4. Vibronic 2D-spectra for the trimer system with a transition dipole geometry of $90^{\circ}$. Panel (a) shows the case of a single vibrational mode per monomer $\left(\omega_{v i b, x}=0.175 \mathrm{eV}\right)$. Including a second monomer vibration with a frequency of $\omega_{v i b, y}=0.120 \mathrm{eV}$ leads to the spectrum in panel (b).

shown in Fig. 5. The monomer spectrum (panel (a)) shows diagonal and cross peaks at energy differences between the vibronic ground state and excited state vibrational levels. ${ }^{49}$ At this point it is already clear that, in the presence of vibrations, the mere existence of cross peaks does not give evidence for excited state electronic coupling. A dimer with a small coupling element will have a very similar spectrum. For larger couplings, as employed here, the neardegeneracy between the excited state vibrational levels is lifted which, depending on the specific characteristics of the system at hand, gives rise to a change in the level structure. This can be seen in Fig. 5 which contains the dimer spectrum in panel (b). As expected, a different vibronic substructure is encountered if compared to the monomer. Also, the redshift (characteristic for J-aggregates) is clearly seen.

The trimer spectrum (panel (c)) is further red-shifted. This reflects the fact that the lowest singly excited electronic level for a dimer occurs at an energy of $\Delta E-J$ whereas, for a trimer, one finds $\Delta E-\sqrt{2} J$. Furthermore, the vibronic sub-structure changes significantly when compared to the dimer. This means that the $2 \mathrm{D}$-spectrum is very sensitive to the aggregate size. We note that, even if rich vibronic features are not detected experimentally due to limited resolution, one still may pick regions of interest which will reflect the spectral changes upon aggregation. The latter process critically depends on

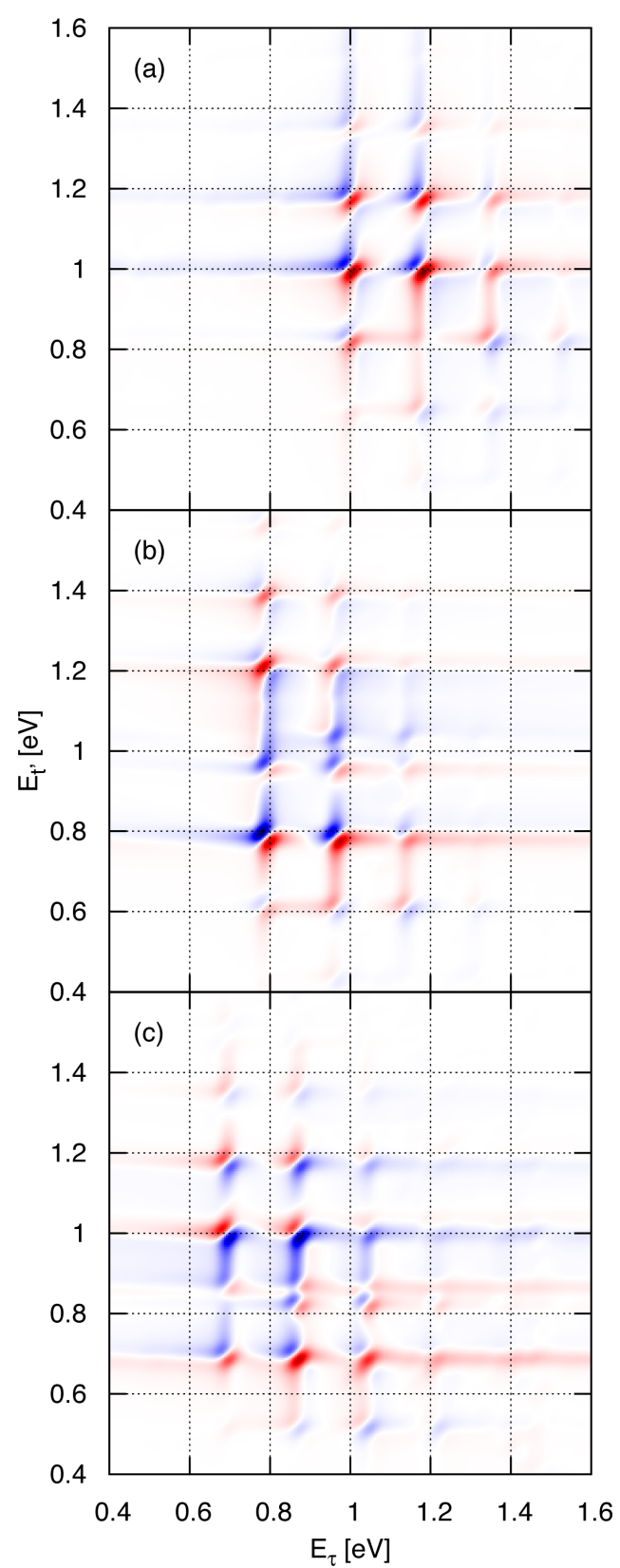

FIG. 5. Comparison of monomer (panel (a)), dimer (panel (b)), and trimer (panel (c)) vibronic 2D-spectra. One monomer vibration is taken into account. The dimer and trimer are in a J-aggregate configuration $\left(\gamma=180^{\circ}\right)$.

temperature. For example, with decreasing temperature it is more likely to encounter larger aggregates. In Fig. 6 we provide an example which illustrates what happens if a sample is cooled. Panel (a) shows a 2D-spectrum which is obtained from a 1:1 mixture of monomers and dimers. The different spectral features can be identified by comparison with the monomer and dimer spectra shown in Fig. 5. With decreasing temperature also trimers are built. This is clearly visible in the spectrum shown in panel (b) of Fig. 6 which results from a 1:1:1 mixture of monomers, dimers, and trimers. Finally, if only dimers and trimers exist (1:1 mixture), the spectrum changes again (panel (c)). For example, the monomer contribution at higher energy $E_{t^{\prime}}$ is then missing. 


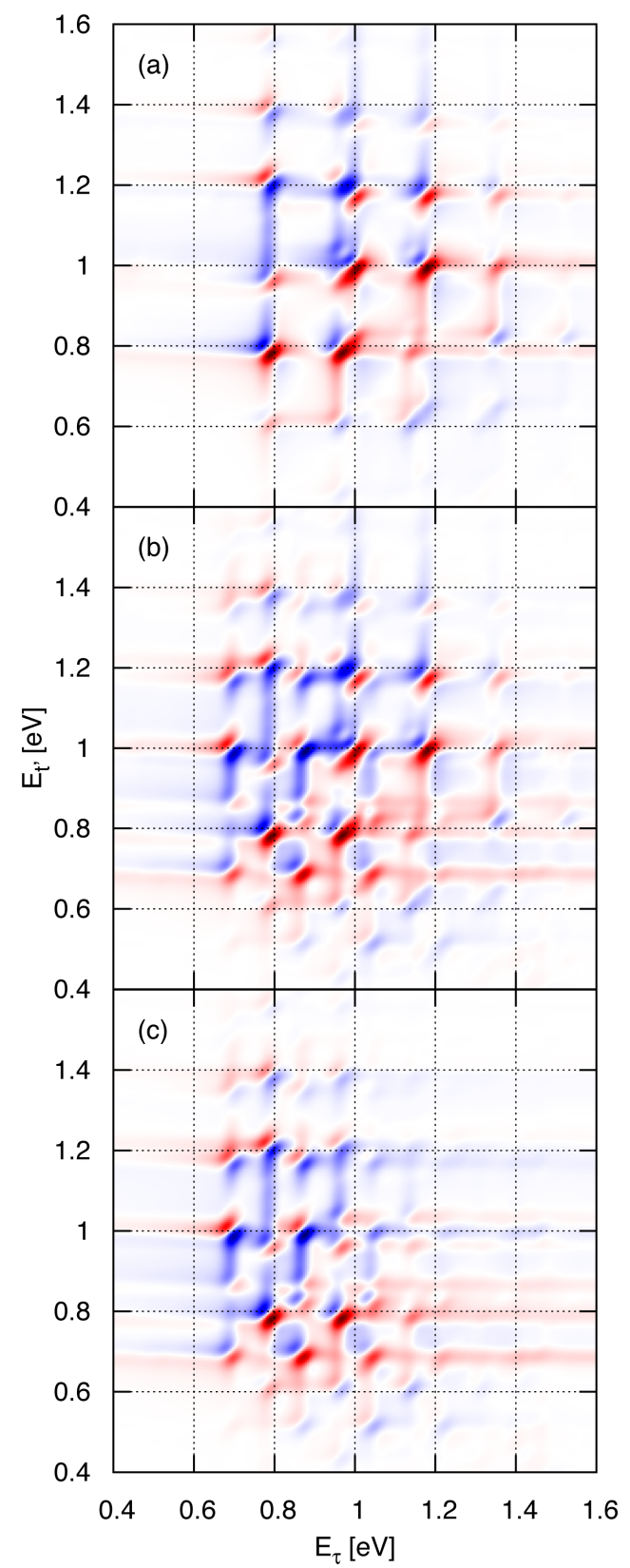

FIG. 6. 2D-spectra resulting from mixtures of different aggregates. Panel (a): 1:1 monomers and dimers; panel (b): 1:1:1 monomers, dimers, and trimers; panel (c): 1:1 dimers and trimers.

\section{SUMMARY}

We calculate 2D vibronic spectra of molecular aggregates by solving the time-dependent Schrödinger equation. For a trimer, this involves 7 coupled electronic states including three and also six vibrational degrees of freedom. The time-propagation is carried through with the computationally effective MCTDH method. Within this approach the timedependent polarization is calculated numerically exactly, and the contribution emitted into the photon-echo phase-matching direction is extracted in repeating the calculation with different phases of the electric fields.

The trimer-spectra for $\mathrm{H}$ - and J-aggregate prototypes exhibit a rich vibronic structure even if only a total number of three (one per monomer) vibrational coordinates are taken into account. Such features can already be seen in the monomer and also the dimer spectrum where the degree of complexity increases with increasing aggregate size. We provide examples of how vibronic 2D-spectra change as a function of the aggregate size. For a J-aggregate, the increasing red shift in going from a monomer to the trimer is reflected in the spectra. Furthermore, vibrationally resolved spectra become more and more structured which is due to the increasing density of vibronic states. The situation is even more dramatic for $\mathrm{H}$-aggregates because even more states are coupled by the fields. The results document how 2D-spectroscopy maps the aggregation in molecular samples. The analysis of the spectra, however, is very challenging. This is mainly due to the vibrational degrees of freedom. Within models consisting of only electronic levels, a monomer exhibits a single diagonal peak. Including vibrations (if only in the simple way used here) already leads to several diagonal and also cross peaks. The potential of optical 2D-spectroscopy lies in its ability to detect electronic couplings which are exemplified by off-diagonal peaks. For a dimer with vibrations and a very small coupling, the $2 \mathrm{D}$-spectrum will resemble the monomer spectrum so that the position of these peaks is not a measure of the coupling but are simply due to vibrations. For stronger couplings, the situation becomes more complicated and our results indicate that only a theory on the vibronic system can help to identify excited state couplings. Of course, going to larger aggregates makes things worse. In calculating 2D-spectra for trimers including vibrations without further approximations on the number of vibrational states, we document systematically how the spectra change. This, to the best of our knowledge, has not been presented before.

To conclude, the spectra show pronounced changes in going from monomers to trimers. We note that the observed redshift is also seen in linear absorption spectra and may be estimated analytically using purely electronic level models. However, the significant changes in the vibrational substructure seen along two energy axis provide a more sensitive tool to extract information about the distribution of aggregate sizes in a mixture, and for this detailed simulations are required to understand the origins of the peaks and assign the spectrum. We hope that our study provides a first step for the interpretation of future experiments.

\section{ACKNOWLEDGMENTS}

Financial support by the DFG within the FOR 1809 and the DAAD is gratefully acknowledged.

${ }^{1}$ E. E. Jelly, Nature 138, 1009 (1936).

${ }^{2}$ G. Scheibe, Angew. Chem. 50, 212 (1937).

${ }^{3}$ E. T. Kobayashi, J-Aggregates (World Scientific, Singapore, 1996).

${ }^{4} \mathrm{H}$. van Amerongen, L. Valkunas, and R. van Grondelle, Photosynthetic Excitons (World Scientific, Singapore, 2000).

${ }^{5}$ J.-L. Brédas, D. Beljonne, V. Coropceanu, and J. Cornil, Chem. Rev. 104, 4971 (2004).

${ }^{6}$ M. R. Wasielewski, Acc. Chem. Res. 42, 1910 (2009).

${ }^{7}$ F. Würthner and K. Meerholz, Chem. Eur. J. 16, 9366 (2010).

${ }^{8}$ M. Kasha, Radiat. Res. 20, 55 (1963).

${ }^{9}$ A. S. Davydov, Theory of Molecular Excitons (Plenum, New York, 1971).

${ }^{10} \mathrm{~V}$. May and O. Kühn, Charge and Energy Transfer Dynamics in Molecular Systems, 3rd ed. (Wiley-VCH, Weinheim, 2011). 
${ }^{11}$ W. T. Simpson and D. L. Peterson, J. Chem. Phys. 26, 588 (1957).

${ }^{12}$ A. Witkowski and W. Moffitt, J. Chem. Phys. 33, 872 (1960).

${ }^{13}$ M. Kasha, H. R. Rawls, and M. A. El-Bayoumi, Pure Appl. Chem. 11, 371 (1965).

${ }^{14}$ R. L. Fulton and M. Gouterman, J. Chem. Phys. 35, 1059 (1961).

${ }^{15}$ R. L. Fulton and M. Gouterman, J. Chem. Phys. 41, 2280 (1964).

${ }^{16} \mathrm{M}$. Schröter, S. Ivanov, J. Schulze, S. Polyutov, Y. Yani, T. Pullerits, and O. Kühn, Phys. Rep. 567, 1 (2015).

${ }^{17}$ W. P. Aue, E. Bartholdi, and R. R. Ernst, J. Chem. Phys. 64, 2229 (1976).

${ }^{18}$ R. R. Ernst, G. Bodenhausen, and A. Wokaun, Principles of Nuclear Magnetic Resonance in One and Two Dimensions (Clarendon Press, Oxford, 1987).

${ }^{19}$ P. Hamm, M. Lim, and R. M. Hochstrasser, J. Phys. Chem. B 102, 6123 (1998).

${ }^{20}$ M. Khalil, N. Demirdöven, and A. Tokmakoff, J. Phys. Chem. A 107, 5258 (2003).

${ }^{21}$ J. Bredenbeck, J. Helbing, C. Kolano, and P. Hamm, ChemPhysChem 8, 1747 (2007).

${ }^{22} \mathrm{M}$. Cho, Two-Dimensional Optical Spectroscopy (CRC Press, Baton Rouge, 2009).

${ }^{23}$ I. J. Finkelstein, J. Zheng, H. Ishikawa, S. Kim, K. Kwak, and M. D. Fayer, Phys. Chem. Chem. Phys. 9, 1533 (2007).

${ }^{24} \mathrm{P}$. Hamm and M. Zanni, Concepts and Methods of $2 \mathrm{D}$ Infrared Spectroscopy (Cambridge University Press, Cambridge, 2011).

${ }^{25}$ J. D. Hybl, A. W. Albrecht, S. M. G. Faeder, and D. M. Jonas, Chem. Phys. Lett. 297, 307 (1998).

${ }^{26}$ P. Tian, D. Keusters, Y. Suzaki, and W. S. Warren, Science 300, 1553 (2003).

${ }^{27}$ M. L. Cowan, J. P. Ogilvie, and R. J. D. Miller, Chem. Phys. Lett. 386, 184 (2004).

${ }^{28}$ T. Brixner, T. Mančal, I. Stiopkin, and G. R. Fleming, J. Chem. Phys. 121, 4221 (2004).

${ }^{29}$ T. Brixner, J. Stenger, H. M. Vaswani, M. Cho, R. E. Blankenship, and G. R. Fleming, Nature 434, 625 (2005).

${ }^{30}$ V. Szöcs, T. Palszegi, V. Lukes, J. Sperling, F. Milota, W. Jakubetz, and H. F. Kauffmann, J. Chem. Phys. 124, 124511 (2006).

${ }^{31}$ G. S. Engel, T. R. Calhoun, E. L. Read, T.-K. Ahn, T. Mančal, Y.-C. Cheng, R. E. Blankenship, and G. R. Fleming, Nature 446, 782 (2007).

${ }^{32}$ A. Nemeth, F. Milota, T. Mančal, V. Lukes, H. F. Kauffmann, and J. Sperling, Chem. Phys. Lett. 459, 94 (2008).

${ }^{33}$ E. L. Read, G. S. Schlau-Cohen, G. S. Engel, J. Wen, R. E. Blankenship, and G. R. Fleming, Biophys. J. 95, 847 (2008).

${ }^{34}$ J. A. Myers, K. L. M. Lewis, F. D. Fuller, P. F. Tekavec, C. F. Yocum, and J. P. Ogilvie, J. Phys. Chem. Lett. 1, 2774 (2010).

${ }^{35}$ E. Collini, C. Wong, K. Wilk, P. Curmi, P. Brumer, and G. D. Scholes, Nature 463, 644 (2010).

${ }^{36}$ M. Kullmann, S. Ruetzel, J. Buback, P. Nuernberger, and T. Brixner, J. Am. Chem. Soc. 133, 13074 (2011).

${ }^{37}$ O. Bixner, V. Lukes, T. Mančal, J. Hauer, F. Milota, M. Fischer, I. Pugliesi, M. Bradler, W. Schmid, E. Riedle, H. F. Kauffmann, and N. Christensson, J. Chem. Phys. 136, 204503 (2012).

${ }^{38}$ T. Mančal, N. Christensson, V. Lukes, F. Milota, O. Bixner, H. F. Kauffmann, and J. Hauer, J. Phys. Chem. Lett. 3, 1497 (2012).

${ }^{39}$ N. Krebs, I. Pugliesi, J. Hauer, and E. Riedle, New J. Phys. 15, 085016 (2013).

${ }^{40}$ C. Consani, G. Auböck, F. van Mourik, and M. Chergui, Science 339, 1586 (2013).

${ }^{41}$ I. Hwang, U. Selig, S. S. Y. Chen, P. E. Shaw, T. Brixner, P. L. Burn, and G. D. Scholes, J. Phys. Chem. A 117, 6270 (2013).

${ }^{42}$ S. Ruetzel, M. Kullmann, J. Buback, P. Nuernberger, and T. Brixner, Phys. Rev. Lett. 110, 148305 (2013).

${ }^{43}$ S. Ruetzel, M. Diekmann, P. Nuernberger, C. Walter, B. Engels, and T. Brixner, Proc. Natl. Acad. Sci. U. S. A. 111, 4764 (2014).

${ }^{44}$ V. Perlik, C. Lincoln, F. Sanda, and J. Hauer, J. Phys. Chem. Lett. 5, 404 (2014).
${ }^{45}$ P. Nuernberger, S. Ruetzel, and T. Brixner, Angew. Chem., Int. Ed. Engl. 54, 11368 (2015).

${ }^{46}$ S. Mukamel, Principles of Nonlinear Optical Spectroscopy (Oxford University Press, New York, 1995).

${ }^{47}$ S. M. G. Faeder and D. M. Jonas, J. Phys. Chem. A 103, 10489 (1999).

${ }^{48}$ S. Mukamel, Annu. Rev. Phys. Chem. 51, 691 (2000).

${ }^{49}$ D. Egorova, M. F. Gelin, and W. Domcke, J. Chem. Phys. 126, 074314 (2007).

${ }^{50}$ M. F. Gelin, D. Egorova, and W. Domcke, Acc. Chem. Res. 42, 1290 (2009).

${ }^{51}$ J. Seibt and A. Eisfeld, J. Chem. Phys. 136, 024109 (2012).

${ }^{52}$ J. Seibt and T. Pullerits, J. Phys. Chem. C 117, 18728 (2013).

${ }^{53}$ T. M. L. Valkunas and D. Abramavicius, Molecular Excitation Dynamics and Relaxation: Quantum Theory and Spectroscopy (Wiley-VCH, Weinheim, 2013).

${ }^{54}$ J. Seibt, K. Renziehausen, D. V. Voronine, and V. Engel, J. Chem. Phys. 130, 134318 (2009).

${ }^{55}$ A. Schubert and V. Engel, J. Chem. Phys. 134, 104304 (2011).

${ }^{56}$ J. Krcmar, F. Gelin, and W. Domcke, Chem. Phys. 422, 53 (2013).

${ }^{57}$ J. Wehner, M. Falge, W. T. Strunz, and V. Engel, J. Chem. Phys. 141, 134306 (2014).

${ }^{58}$ J. Albert, M. Falge, M. Keß, J. Wehner, P. Zhang, A. Eiseld, and V. Engel, J. Chem. Phys. 142, 212440 (2015).

${ }^{59}$ T. Holstein, Ann. Phys. 8, 325 (1959).

${ }^{60}$ F. C. Spano, Acc. Chem. Res. 43, 429 (2010).

${ }^{61}$ R. F. Fink, J. Seibt, V. Engel, M. Renz, M. Kaupp, S. Lochbrunner, H.-M. Zhao, J. Pfister, F. Würthner, and B. Engels, J. Am. Chem. Soc. 130, 12858 (2008).

${ }^{62}$ A. Schubert, M. Falge, M. Kess, V. Settels, S. Lochbrunner, W. T. Strunz, F. Würthner, B. Engels, and V. Engel, J. Phys. Chem. A 118, 1403 (2014).

${ }^{63}$ J. Roden, A. Eisfeld, M. Dvorak, O. Bünemann, and F. Stienkemeier, J. Chem. Phys. 134, 054907 (2011).

${ }^{64}$ H.-D. Meyer, U. Manthe, and L. S. Cederbaum, Chem. Phys. Lett. 165, 73 (1990).

${ }^{65}$ M. H. Beck, A. Jäckle, G. A. Worth, and H.-D. Meyer, Phys. Rep. 324, 1 (2000).

${ }^{66}$ Multidimensional Quantum Dynamics: MCTDH Theory and Applications, edited by H.-D. Meyer, F. Gatti, and G. A. Worth (Wiley-VCH, Weinheim, 2009).

${ }^{67}$ H.-S. Tan, J. Chem. Phys. 129, 124501 (2006).

${ }^{68}$ P. F. Tekavec, G. A. Lott, and A. H. Marcus, J. Chem. Phys. 127, 214307 (2007).

${ }^{69}$ L. Seidner, G. Stock, and W. Domcke, J. Chem. Phys. 103, 3998 (1995).

${ }^{70}$ S. Meyer and V. Engel, Appl. Phys. B 71, 293 (2000).

${ }^{71}$ M. F. Gelin, D. Egorova, and W. Domcke, J. Chem. Phys. 123, 164112 (2005).

${ }^{72}$ Z. Chen, V. Stepanenko, V. Dehm, P. Prins, L. Siebbeles, J. Seibt, P. Marquetand, V. Engel, and F. Würthner, Chem. Eur. J. 13, 436 (2007).

${ }^{73}$ J. Seibt, T. Winkler, K. Renziehausen, V. Dehm, F. Würthner, and V. Engel, J. Phys. Chem. A 113, 13475 (2009).

${ }^{74}$ J. Seibt, V. Dehm, F. Würthner, and V. Engel, J. Chem. Phys. 128, 204303 (2008).

${ }^{75}$ P. Kjellberg, B. Brüggemann, and T. Pullerits, Phys. Rev. B 74, 024303 (2006).

${ }^{76}$ J. Seibt, P. Marquetand, V. Engel, Z. Chen, V. Dehm, and F. Würthner, Chem. Phys. 328, 354 (2006).

${ }^{77}$ C. Brüning, K. Renziehausen, and V. Engel, J. Chem. Phys. 139, 054303 (2013).

${ }^{78}$ A. Eisfeld, L. Braun, W. T. Strunz, J. S. Briggs, J. Beck, and V. Engel, J. Chem. Phys. 122, 134103 (2005).

${ }^{79}$ J. Seibt and V. Engel, Chem. Phys. 347, 120 (2008).

${ }^{80}$ G. A. Worth, M. H. Beck, A. Jäckle, and H.-D. Meyer, The MCTDH Package, version 8.2 (2000); H.-D. Meyer, version 8.3 (2002) and version 8.4 (2007). See http://www.pci.uni-heidelberg.de/tc/usr/mctdh/.

${ }^{81} \mathrm{~W}$. Kutzelnigg, Einführung in die Theoretische Chemie, Band $2(\mathrm{VCH}$, Weinheim, 1994).

${ }^{82}$ D. Egorova, J. Chem. Phys. 140, 034314 (2014). 